\title{
Photon splitting in strongly magnetized medium with tak- ing into account positronium influence
}

\author{
Anikin R.A. ${ }^{1, *}$, Chistyakov M.V. ${ }^{2, * *}$, Rumyantsev D.A. ${ }^{2, * * *}$, and Shlenev D.M. ${ }^{1, * * * *}$ \\ ${ }^{1}$ Division of Physics, Yaroslavl Higher Military School of Air Defense, Moscow Prospect 28, 150001 \\ Yaroslavl, Russian Federation \\ ${ }^{2}$ Division of Theoretical Physics, Department of Physics, Yaroslavl State P. G. Demidov University, \\ Sovietskaya 14, 150000 Yaroslavl, Russian Federation
}

\begin{abstract}
The process of the photon splitting, $\gamma \rightarrow \gamma \gamma$, is investigated in strongly magnetized vacuum with taking into account positronium influence. The dispersion properties of photons and the new polarization selection rules are obtained. The absorption rate of the leading photon splitting channels are calculated with taking account of the photon dispersion and wave function renormalization.
\end{abstract}

\section{Introduction}

The process of photon splitting into two photons, which is forbidden in vacuum by charge conjugation symmetry of QED known as Furry's theorem, becomes allowed in the presence of external magnetic field. This process was investigated in numerous papers because of it's possible applications in astrophysics (for detailed review see pioneer work [1] and recent paper [2]). It may play an important role in explanation of peculiarities in the gamma-ray spectra of some radio pulsars, the radio quiescence of soft gamma repeaters (SGRs) and anomalous X-ray pulsars (AXPs) and mechanisms of the SGR burst [3]. Note, that the scale of the magnetic field of the above objects may reach the critical value $B_{c r}=m^{2} / e \simeq 4.41 \times$ $10^{13} \mathrm{G}^{1}$ or even exceed it [4].

Positronium influence on dispertion properties of photon was investigated in [5] on example of process $v \rightarrow v \gamma$ in strong magnetic field. Analysis shows that positronium effects may significally change kinemaitics and probability of this process by altering dispertion properties of photon.

Therefore, it's interesting to calculate the probability of photon splitting process in strong magnetic field with taking into account variations of dispertion properties and kinematics due to positronium influence.

\footnotetext{
*e-mail: anik-roman@mail.ru

**e-mail: mch@uniyar.ac.ru

***e-mail: rda@uniyar.ac.ru

****e-mail: allen_caleb@rambler.ru

${ }^{1}$ We use the natural units $c=\hbar=k=1, m$ is the electron mass, $e>0$ is the elementary charge.
} 


\section{Photon dispersion with taking into account positronium influence}

We begin to consider the process $\gamma \rightarrow \gamma \gamma$ with investigation of the photon dispersion properties. The propagation of an electromagnetic radiation in any active medium is conveniently described in terms of normal modes (eigenmodes). In turn, the polarization and dispersion properties of the normal modes are defined by the eigenvectors and eigenvalues of the polarization operator $\mathcal{P}_{\alpha \beta}$, respectively [6].

Hereafter we use the following notations: the four-vectors with the indices $\perp$ and $\|$ belong to the Euclidean $\{1,2\}$ subspace and the Minkowski $\{0,3\}$ subspace correspondingly. Then for arbitrary 4-vectors $A_{\mu}, B_{\mu}$ one has

$$
\begin{aligned}
& A_{\perp}^{\mu}=\left(0, A_{1}, A_{2}, 0\right), \quad A_{\|}^{\mu}=\left(A_{0}, 0,0, A_{3}\right), \\
& (A B)_{\perp}=(A \Lambda B)=A_{1} B_{1}+A_{2} B_{2}, \\
& (A B)_{\|}=(A \widetilde{\Lambda} B)=A_{0} B_{0}-A_{3} B_{3},
\end{aligned}
$$

where the matrices $\Lambda_{\mu v}=(\varphi \varphi)_{\mu v}, \widetilde{\Lambda}_{\mu v}=(\tilde{\varphi} \tilde{\varphi})_{\mu v}$ are constructed with the dimensionless tensor of the external magnetic field, $\varphi_{\mu \nu}=F_{\mu \nu} / B$, and the dual tensor, $\tilde{\varphi}_{\mu \nu}=\frac{1}{2} \varepsilon_{\mu \nu \rho \sigma} \varphi^{\rho \sigma}$. The matrices $\Lambda_{\mu \nu}$ and $\widetilde{\Lambda}_{\mu v}$ are connected by the relation $\widetilde{\Lambda}^{\mu v}-\Lambda^{\mu v}=g^{\mu v}=\operatorname{diag}(1,-1,-1,-1)$, and play the roles of the metric tensors in the perpendicular $(\perp)$ and the parallel $(\|)$ subspaces respectively.

It had been shown in [5] that positronium influence leaves physical polarisation eigenvectors ${ }^{2}$ just the same as in magnetized vacuum without positronium contribution.

$$
\varepsilon_{\alpha}^{(1)}(q)=\frac{(q \varphi)_{\alpha}}{\sqrt{q_{\perp}^{2}}}, \quad \varepsilon_{\alpha}^{(2)}(q)=\frac{(q \tilde{\varphi})_{\alpha}}{\sqrt{q_{\|}^{2}}} .
$$

Eigenvalue for photon of mode 1 also remains unchanged from the case of magnetized vacuum without positronium contribution. This leads to almost vacuum dispertion law for photon of the first mode.

$$
\varkappa^{(1)}=-\frac{\alpha}{3 \pi} q_{\perp}^{2}
$$

Taking into account of positronium influence modifies the eigenvalue for photon of mode 2 with respect to the case of magnetized vacuum without positronium contribution.

$$
\begin{aligned}
& \varkappa^{(2)} \simeq-\frac{2 \alpha}{\pi} e B\left[H(x)+\frac{\pi v x}{1-v^{2}-x}\right] \\
& x=\frac{q_{\|}^{2}}{4 m^{2}}, \quad y=\frac{q_{\perp}^{2}}{4 m^{2}}, \quad H(x)=\frac{1}{\sqrt{x(1-x)}} \arctan \sqrt{\frac{x}{1-x}}-1, \\
& v=\frac{\alpha}{2}\left[\ln (4.5 u-2.44 \ln (\ln (0.15 u))], \quad u=32\left(\frac{e B}{\alpha q_{\perp}^{2}}\right)^{2} .\right.
\end{aligned}
$$

Dispertion law for photon of the mode 2 significally differs from the case of magnetized vacuum case without positronium contribution. Single branch splits in two, upper converges to dispertion of photon with unbound electron-positron pair in loop, lower - to positronium spectral line.

\footnotetext{
${ }^{2}$ Symbols 1 and 2 correspond to the $\|$ and $\perp$ polarizations in a pure magnetic field in Adler's notation [1].
} 
In Fig. 1, the dispersion law of the mode 2 photon is depicted as the solution of the equation

$$
q^{2}-\varkappa^{(2)}=0
$$

It follows from Eq. (4a) that the eigenvalue of the polarization operator $\varkappa^{(2)}$ becomes large near the electron-positron pair production threshold (see Fig. 1). This means that the renormalization of the wave function for a photon of this polarization should be taken into account:

$$
\varepsilon_{\alpha}^{(2)}(q) \rightarrow \varepsilon_{\alpha}^{(2)}(q) \sqrt{Z_{2}}, \quad Z_{2}^{-1}=1-\frac{\partial \varkappa^{(2)}(q)}{\partial \omega^{2}} .
$$

Modifications of the dispertion properties due to positronium influence define new selection rules of photon splitting process. The existence of kinematic area with $q^{2}>0$ for the upper branch of photon of the mode 2 opens new photon splitting channel $\gamma_{2} \rightarrow \gamma_{1} \gamma_{1}$, which was considered forbidden in the magnetic field, while the splitting channels $\gamma_{1} \rightarrow \gamma_{2} \gamma_{2}$ and $\gamma_{1} \rightarrow \gamma_{1} \gamma_{2}$ allowed in case of magnetized vacuum without positronium contribution, are forbidden in this area. In the region $q^{2}<0$, polarization selection rules are the same ones as in the magnetized vacuum [1].

\section{The probability of the photon splitting}

Photon absorption rate due to the photon splitting can be defined in the following way:

$$
W_{\lambda \rightarrow \lambda^{\prime} \lambda^{\prime \prime}}=\frac{g_{\lambda^{\prime} \lambda^{\prime \prime}}}{32 \pi^{2} \omega_{\lambda}} \int\left|\mathcal{M}_{\lambda \rightarrow \lambda^{\prime} \lambda^{\prime \prime}}\right|^{2} Z_{\lambda} Z_{\lambda^{\prime}} Z_{\lambda^{\prime \prime}} \delta\left(\omega_{\lambda}(\mathbf{k})-\omega_{\lambda^{\prime}}\left(\mathbf{k}-\mathbf{k}^{\prime \prime}\right)-\omega_{\lambda^{\prime \prime}}\left(\mathbf{k}^{\prime \prime}\right)\right) \frac{d^{3} k^{\prime \prime}}{\omega_{\lambda^{\prime}} \omega_{\lambda^{\prime \prime}}}
$$

where $\lambda$ - polarisation mode of initial photon, $\lambda^{\prime}$ and $\lambda^{\prime \prime}$ - polarisation modes of final photons, the factor $g_{\lambda^{\prime} \lambda^{\prime \prime}}=1-(1 / 2) \delta_{\lambda^{\prime} \lambda^{\prime \prime}}$ is inserted to account for the possible identity of the final photons. Here $q_{\alpha}=\left(\omega_{\lambda}, \mathbf{k}\right)$ - four-momentum of initial photon, $q_{\alpha}^{\prime}=\left(\omega_{\lambda^{\prime}}, \mathbf{k}^{\prime}\right)$ and $q_{\alpha}^{\prime \prime}=$ $\left(\omega_{\lambda^{\prime \prime}}, \mathbf{k}^{\prime \prime}\right)$ - four-momenta of final photons.

Amplitudes of the photon splitting process in magnetized vacuum without positronium contribution $\mathcal{M}_{\lambda \rightarrow \lambda^{\prime} \lambda^{\prime \prime}}$ where obtained earlier in [7]. They can be presented as follows:

$$
\begin{aligned}
& \mathcal{M}_{1 \rightarrow 12}=-i 4 \pi\left(\frac{\alpha}{\pi}\right)^{3 / 2} \frac{\left(q^{\prime} \varphi q^{\prime \prime}\right)\left(q^{\prime} \tilde{\varphi} q^{\prime \prime}\right)}{\left[q_{\perp}^{2} q_{\perp}^{\prime 2} q_{\|}^{\prime \prime 2}\right]^{1 / 2}} H\left(\frac{q_{\|}^{\prime \prime 2}}{4 m^{2}}\right), \\
& \mathcal{M}_{1 \rightarrow 22}=-i 4 \pi\left(\frac{\alpha}{\pi}\right)^{3 / 2} \frac{\left(q^{\prime} q^{\prime \prime}\right)_{\|}}{\left[q_{\perp}^{2} q_{\|}^{\prime 2} q_{\|}^{\prime \prime 2}\right]^{1 / 2}}\left[\left(q q^{\prime \prime}\right)_{\perp} H\left(\frac{q_{\|}^{\prime 2}}{4 m^{2}}\right)+\left(q q^{\prime}\right)_{\perp} H\left(\frac{q_{\|}^{\prime \prime 2}}{4 m^{2}}\right)\right], \\
& \mathcal{M}_{2 \rightarrow 11}=\mathcal{M}_{1 \rightarrow 12}\left(q \leftrightarrow q^{\prime \prime}\right)
\end{aligned}
$$

Absorption rates of the channels $\gamma_{1} \rightarrow \gamma_{1} \gamma_{2}$ and $\gamma_{1} \rightarrow \gamma_{2} \gamma_{2}$ were calculated numerically for the magnetic field strenght $B=200 B_{c r}$. In Fig. 2 and Fig. 3 they are depicted as functions of the initial photon energy.

Analytical expression for the absorption rate of the channel $\gamma_{2} \rightarrow \gamma_{1} \gamma_{1}$ could be obtained from [2] and has the following form: 


$$
\begin{array}{r}
W_{2 \rightarrow 11}=\frac{\alpha^{3}}{8 \pi^{2}} Z_{2} H\left(\frac{q_{\|}^{\prime \prime 2}}{4 m^{2}}\right) \frac{q_{\perp}^{2}}{\omega} \mathcal{F}\left(\sqrt{\frac{q_{\|}^{2}}{q_{\perp}^{2}}}\right) \Theta\left(q^{2}\right), \\
\mathcal{F}(z)=2 \ln z-1+z^{-2}
\end{array}
$$

\section{Conclusion}

The obtained results show that positronium influence modifies the polarization selection rules in comparison with a case of magnetized vacuum without taking into account of positronium influence. In particular, the splitting channel $\gamma_{2} \rightarrow \gamma_{1} \gamma_{1}$, which was considered forbidden, is allowed.

In the case of the channels $\gamma_{1} \rightarrow \gamma_{1} \gamma_{2}$ and $\gamma_{1} \rightarrow \gamma_{2} \gamma_{2}$ positronium has an ambiguous influence on absorption rate. It splits one dispertion branch for photon of the 2 mode in two, but also decreases phase space for these processes. Existing area of $q^{2}<0$ implies additional constraints on probability of process $\gamma_{1} \rightarrow \gamma_{2} \gamma_{2}$. As a result, absorption rate due to channels $\gamma_{1} \rightarrow \gamma_{1} \gamma_{2}$ and $\gamma_{1} \rightarrow \gamma_{2} \gamma_{2}$ becomes suppressed in comparison with a case of magnetic field without positronium contribution.

\section{Acknowledgements}

We are grateful to A. V. Kuznetsov for useful discussions and valuable remarks. D. S. expresses his deep gratitude to the organizers of the Seminar "Quarks-2018" for warm hospitality.

The paper is partially supported by the YSU Initiative Scientific Research Activity (Project No. AAAA-A16-116070610023-3).

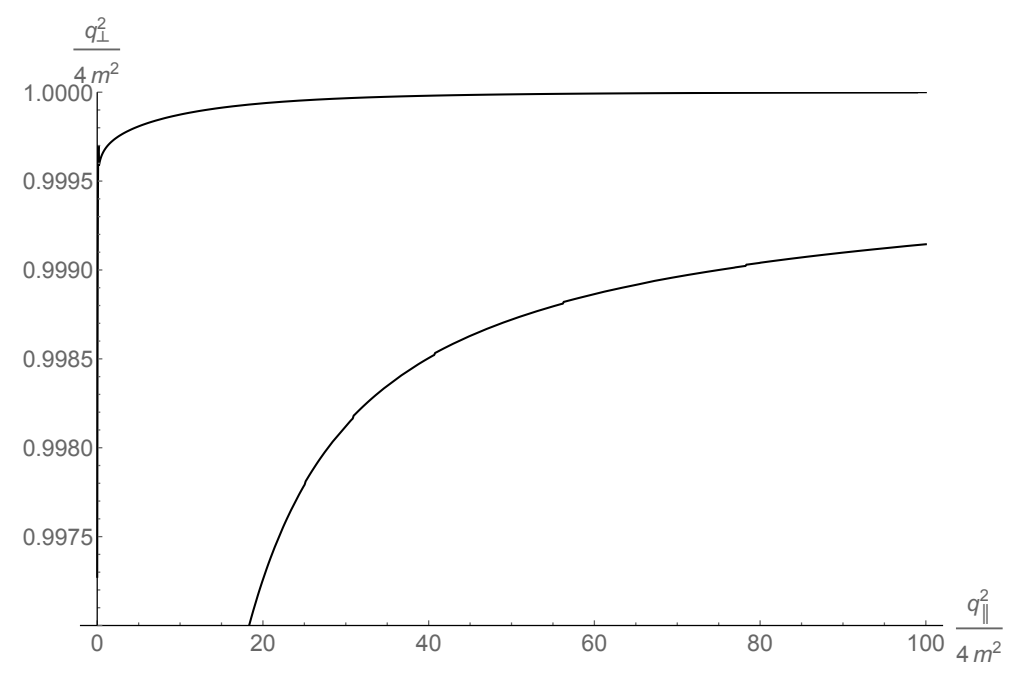

Figure 1. The photon dispersion of photon of the 2 mode in a strong magnetic field $\left(B / B_{c r}=200\right)$. 


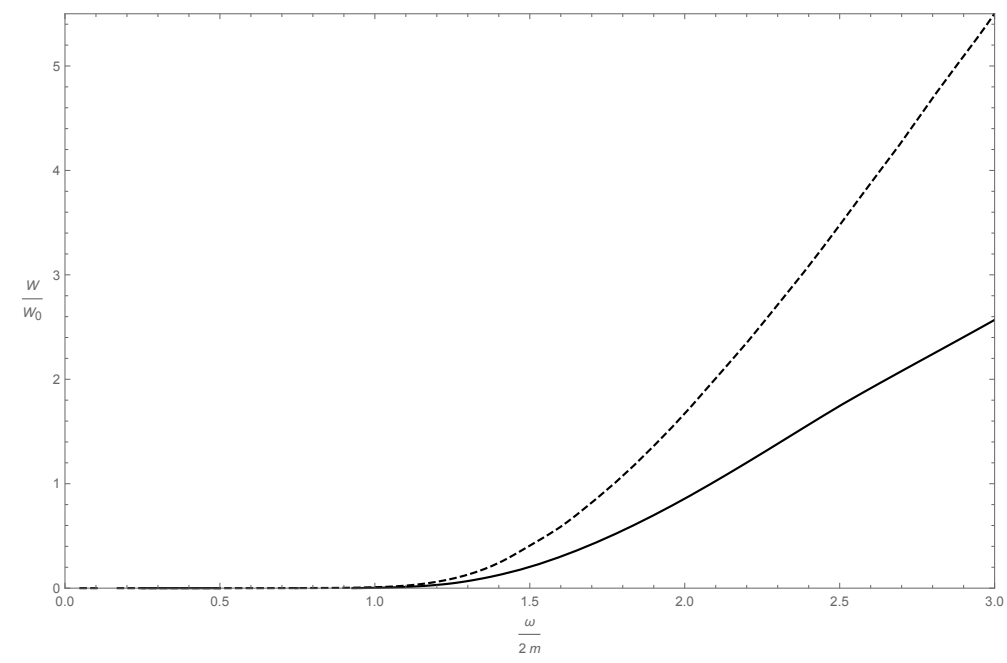

Figure 2. The photon absorption rate due to the channel $\gamma_{1} \rightarrow \gamma_{1} \gamma_{2}$ in a strong magnetic field $\left(B / B_{e}=200\right)$. Dashed line corresponds to the probability in magnetized vacuum without positronium influence [7]. Here $W_{0}=(\alpha / \pi)^{3} \mathrm{~m} \simeq 3.25 \cdot 10^{2} \mathrm{~cm}^{-1}$.

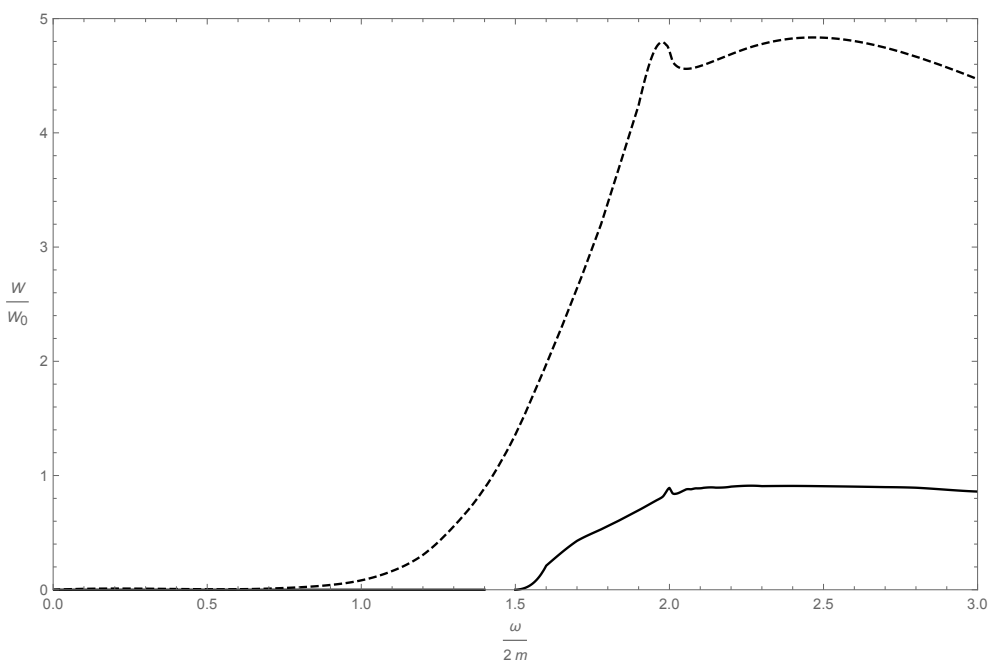

Figure 3. The photon absorption rate due to the channel $\gamma_{1} \rightarrow \gamma_{2} \gamma_{2}$ in a strong magnetic field $\left(B / B_{e}=200\right)$. Dashed line corresponds to the probability in magnetized vacuum without positronium influence [7]. Here $W_{0}=(\alpha / \pi)^{3} \mathrm{~m} \simeq 3.25 \cdot 10^{2} \mathrm{~cm}^{-1}$.

\section{References}

[1] S. L. Adler, Ann. Phys. (N.Y.) 67, 599 (1971).

[2] D. A. Rumyantsev, M. V. Chistyakov, N. S. Stus', Phys. Rev. D 86, 043007 (2012).

[3] D. Lai, Rev. Mod. Phys. 73, 629 (2001).

[4] R. C. Duncan, C. Thompson, Astrophys. J. 392, L9 (1992).

[5] R. A. Anikin, N. V. Mikheev, Journ. of Exp. and Theor. Phys. 115, 411 (2012). 
[6] A. E. Shabad, Tr. Fiz. Inst. Akad. Nauk SSSR 192, 5 (1988).

[7] M. V. Chistyakov, A. V. Kuznetsov, N. V. Mikheev, Phys.Lett. B434, 67 (1998);

A. V. Kuznetsov, N. V. Mikheev, M. V. Chistyakov, Yad. Fiz. 62, 1638 (1999) [Phys. At. Nucl. 62, 1535 (1999)]. 